\title{
Dual-channel microarray sensor system for lung cancer-related volatile organic compounds identification in exhaled breath
}

\author{
Yonghong Xu, Jihong Jiang, Huimin Bu, Pengcheng Zhu, Jiebang Jiang, \\ Yejiao Wu, Rongpeng $\mathrm{Li}^{*}$
}

The Key Laboratory of Biotechnology for Medicinal Plants of Jiangsu Province, Jiangsu Normal University, Xuzhou, 221116, China

*Corresponding Author: Prof. Rongpeng Li

\author{
The Key Laboratory of Biotechnology for Medicinal Plants of \\ Jiangsu Province and School of Life Sciences, \\ Jiangsu Normal University,
}

Xuzhou, 221116,

P.R. China

Tel: +86-516-83403515

Fax: +86-516-83403515

E-mail address: lirongpeng@jsnu.edu.cn (R.P. Li)

xyh8810@126.com (Y.H. Xu)

jhjiang669@126.com (J.H. Jiang) 
Abstract: Lung cancer remains the leading cancer killer worldwide. Early diagnosis can effectively increase the patient cure rate but existing diagnostic methods limit early lung cancer diagnosis. Therefore, development of a simple but efficient lung cancer screening method is important to improvement of both the diagnosis rate and the survival rate of lung cancer patients. In this study, ten photosensitive materials with high sensitivity and high specificity were screened accurately to construct a microarray sensor that can rapidly identify six types of lung cancer biomarkers in exhaled breath. Results from hierarchical cluster analysis (HCA), principal component analysis (PCA) and difference maps showed that the classification of the analytes agreed with structure similarity laws. The detection results from parallel experiments and structurally similar analytes, in turn, cluster into a group; the fingerprints of the different analytes have specific response regions. The well-screened sensor chip fabrication workload and cost were both reduced by approximately two thirds, while the microfluidic device sensitivity and stability increased by approximately 1.3 times their corresponding values before optimization. The dualchannel device also offers real-time contrast detection and synchronous parallel detection functions and has potential application prospects for use in extensive screening of high-risk populations for lung cancer.

Keyword: Dual-channel sensor; Efficient capture; Lung cancer biomarker; Lung cancer screening 


\section{Introduction}

Lung cancer is a major disease that poses a serious threat to human health and life. Global cancer statistics data show that there are approximately 18.1 million new cases and 9.6 million deaths from cancer worldwide annually, and the morbidity and mortality rates of lung cancer are the highest among all cancers [1]. Lung cancer has a long incubation period, and if the lifestyles, diets and exercise habits of patients can be consciously improved within this latent period, it may be possible to reverse or prevent lung cancer. However, because there are no obvious symptoms of lung cancer in the early stages, the general public's awareness of prevention measures is poor; this, along with a lack of relevant screening, means that patients often miss the best time for treatment of the disease [2]. Therefore, early screening and diagnosis of lung cancer is both the key link to a cure and the most important bottleneck in the path toward that cure. Design and development of detection methods that can detect the hidden dangers of such a serious disease during the early clinical stage and even during the incubation period of lung cancer is of considerable theoretical significance and practical value. While traditional lung cancer diagnosis techniques, including imaging-based examinations, pathological examinations and microscopic examinations, can improve the survival rates of patients to some extent and reduce the mortality rates of high-risk groups, they are still limited by trauma, high false negatives, high cost and other factors [3, 4].

When a tumor grows in the body, the tumor cells will generate specific substances. These substances are then released into the blood and exchanged in the lungs, thus presenting measurable highly expressed components in the patient's expiration. This indicates the feasibility of expiration-based detection in the breath of lung cancer patients [5]. Pauling et al. first reported that the expiration from an average human contains approximately 250 chemical components [6], and, since then, thousands of these components have been identified. There are not many diseaserelated substances among these components and the relevant concentration is generally low, at around the parts-per-trillion (ppt) level. Even so, the changes in the components and the levels of the trace volatile organic compounds (VOCs) in lung cancer patients show significant differences when compared with those of healthy people, while they also still reflect the metabolism of the lung cancer cells and thus allow the stage of the illness to be determined [7-11]. In breath-based testing of lung cancer [12], while commonly used large instrument analysis technology (e.g., gas 
chromatography (GC), GC-mass spectrometry (GC-MS)) is commercially available, it is a timeconsuming and high-cost approach [13]. Acute olfactory recognition methods can eliminate interference to detect the specific smell of lung cancer accurately, but the special sniffers required must be trained for a long time [14]. Bionic detection technology (electronic noses) can distinguish and identify large numbers of gases, is noninvasive, and offers real-time, convenient and efficient operation. The electrochemical sensing method of this type that has attracted most widespread attention is highly sensitive and low in cost, but still faces challenges in terms of selectivity and anti-interference characteristics [15]; however, the photochemical sensing method based on the cross response is intuitive, fast, safe and efficient, and is thus more suitable for extensive lung cancer screening of high-risk populations [16, 17].

Suslick et al. first proposed the concept of the visual sensor array in 2000 [18], and based on this concept, analysis and recognition of the substances in exhalation that originate from lung cancer were realized [19]; this effectively promoted the diversification and development of lung cancer breath diagnosis. In our previous study, a $6 \times 6$ visualized sensor array was constructed to detect specific lung cancer markers in an exploratory manner [20], and some advances have been made in the lung cancer screening field. However, because of the complexity of the components of the clinical breath samples and the low content levels of the disease-related substances, it is easy for interference signals to be produced and for signals to be missed, so it is necessary to remove impurities and enrichments before detection. Simultaneously, the 36 primarily selected sensing materials did not all have recognition functions on the samples, which means that it is necessary to reduce the detection costs and the time consumed in chip preparation by trimming the sensing materials. To provide further improvements in the accuracy, sensitivity and recognition capability of the detection system in this study, an "adsorption-thermal desorption-dual channel sensing system" will be constructed independently. Simultaneously, based on the results of previous studies, the sensing materials were screened accurately for the molecular structures of lung cancer markers to aid in optimization and fabrication of microarray sensors.

\section{Materials and methods}

\subsection{Materials and chemicals}

The VOCs and the sensitive materials were supplied by Sigma-Aldrich (St. Louis, MO, USA). The porous hydrophobic polyvinylidene fluoride (PVDF) membrane (pore diameter: 0.45 
$\mu \mathrm{m})$ was bought from Millipore Co. Ltd. (Bedford, MA, USA). The quartz capillary (inner diameter: $0.3 \mathrm{~mm}$ ) and the 10L Krinkle Tedalr were purchased from Lekang Reagent Supplies (Xuzhou, China). 99.999\% $\mathrm{N}_{2}$ was bought from the Jiacheng Experimental Instrument business department (Xuzhou, China). A Tenax Ta $200 \mathrm{mg}$ stainless steel adsorption tube and an ATDS3400B low-temperature secondary thermal desorption apparatus were bought from Boli Instrument Co. Ltd. (Shanghai, China). The ultra-pure water was generated using a Millipore Direct-Q Water system (Molsheim, France).

\subsection{Precise selection method of sensitive materials}

When targeting the molecular structures of lung cancer markers contained in exhalation, accurate screening requires the center sites of the sensitive materials to bind tightly to the analytes and generate strong interactions; simultaneously, color changes in the sensing units are caused by the good chromophores and the high cross-response efficiency of the sensitive materials. When using the above screening method, and based on a previous study, three types of sensitive material (Fig. 1) were selected: a response material containing metal ions that could provide electrons or metal coordination sites based on a Lewis acid-base reaction, such as tetraphenyl metal porphyrin (where the metal is zinc, iron, or manganese); a chromotropic dye based on a zwitterionic solvent or gas that would respond to local polar molecules, such as Nile red or disperse orange; and a pH indicator based on Brønsted-Lowry acid-base theory that uses proton acidity or hydrogen bond binding, such as bromocresol green, bromothymol blue, cresol red, brilliant yellow and chlorphenol red. Therefore, when compared with methods that depend on physical adsorption alone, the $2 \times 5$ visual sensor array that was established using 10 sensitive materials (Tab.1) provides greater interaction between the sensitive materials and the analytes. This method can reduce detection costs and workloads, and guarantees the detection efficiency of the sensor array.

\subsection{Microarray chip preparation method}

First, a hydrophobic PVDF membrane was cut into smaller membranes with dimensions of $8 \times 16 \mathrm{~mm}$, which were then placed into a die. A sensitive material solution (approximately $0.1 \mu \mathrm{L}$ ) was dropped using a quartz capillary tube under a $\mathrm{N}_{2}$ protective atmosphere and smeared over the PVDF membrane until the solution spread into a uniformly-sized dot (diameter $\approx 2 \mathrm{~mm}$ ). The material was adhered perfectly on the membrane using microcontact technology to prepare the $2 \times 5$ array chip. The prepared array chip was dried and stored in the N2 atmosphere while also 
being kept out of the light.

\subsection{Calculation methods for RSD and $\mathrm{V}_{\mathrm{i}}$}

The calculation formulas for the relative standard deviation (RSD) and the detection volume of the saturated vapor $\left(\mathrm{V}_{\mathrm{i}}\right)$ are shown in Table 1. The RSD equation is given as follows: $R S D=\frac{S}{\bar{x}} \times 100 \%$, where $S$ is equal to the standard deviation and is determined using the equation $S=\sqrt{\frac{\sum_{i=1}^{n}\left(x_{i}-\bar{x}\right)^{2}}{n-1}}$, and $\bar{X}$ is equal to the mean. A lower percentage in these results indicates greater stability in the data set. $\mathrm{V}_{\mathrm{i}}$ is calculated using the Antoine equation, which relates the vapor pressure to the temperature.

\subsection{Recognition method of sensor}

In this study, the smell is identified by collecting color change information before and after the chemical reaction between the sensitive materials and the VOCs. In the dual-channel microarray sensing system (Fig. 2), a light-emitting diode (LED) and a complementary metaloxide-semiconductor (CMOS) camera combine to convert the chemical reaction signal into a light signal (Fig. 2(a)-(c)), and the red (R), green $(\mathrm{G})$, and blue (B) values are collected from each sensing element (Fig. 2(d)). Visual difference maps can then be obtained using the relative values of RGB change before and after the reactions between each sensor element (Fig. 2(e)-(f)) and the analyte, as illustrated in Fig. 2(g). The image signals and data signals are generated by color changes in the sensing unit that result from the reaction of the photosensitive materials and the analytes; the output signals are then recognized and judged using a pattern recognition system.

\section{Results}

\subsection{Fabrication of dual-channel sensor}

A dual-channel sensor was independently designed and constructed, and its branch channels were aligned with each of the rows of sensitive materials (Fig. 3(c)) to ensure that the detection object and the sensitive material would make contact and react adequately; when compared with the single channel sensing method, it is easier to implement real-time contrast detection and synchronous parallel detection of test sets of "lung cancer clinical breath specimen and control groups," "clinical breath specimen and atmosphere," and "lung cancer clinical breath specimen and nitrogen," with improved detection sensitivity and speed. In this study, soft lithography technology was used to fabricate the dual-channel microstructure of the sensor, which has 
appropriate surface tension and is conducive to detection of gas that is slowly passing through the structure. Simultaneously, the vertical grinding method, the mosaic method and other methods are combined to fabricate an organic glass sensing model and a light-proof box to ensure the airtightness of the instrument.

\subsection{Perfection of the sensor system}

To improve its accuracy, precision and detection efficiency, the sensor system was further improved during this study (Fig. 3). The mixed expired air samples were concentrated using an enrichment tube that was equipped with Tenax Ta $200 \mathrm{mg}$ (Fig. 3(a)). After thermal desorption (Fig. 3(b)), the VOCs pass over the self-made dual-channel microarray chip (Fig. 3(c)) and react with the sensitive material on the chip. This method can avoid false signals and missed signals effectively and is intended to provide an important guarantee and a strategy for effective detection of the clinical breath samples in the later stages.

\subsection{Discrimination of VOCs}

VOC-saturated vapor was extracted using a microsyringe and was then injected into a bag filled with $\mathrm{N}_{2}(10 \mathrm{~L})$ and connected to the dual-channel microarray sensor to realize $0.6 \mathrm{~L} / \mathrm{min}$ constant velocity when driven by a pump. VOCs were detected at four different concentrations, and each experiment was repeated five times. The detection temperature was controlled at $26^{\circ} \mathrm{C}$, and the relative humidity was $32 \%$. The response time was $7 \mathrm{~min}$. Information including the calculated and measured test results for the $10 \mathrm{ppm}$ VOCs are shown in Table 2; the RSD within the control group is $0.1 \%$, thus indicating that the self-built sensor shows stable performance. The RSDs of all the analytes are within the $1.7 \%$ to $2.9 \%$ range (i.e., $<3 \%$ ), thus indicating the good repeatability of the proposed method.

\subsection{Fingerprint analysis of VOCs}

Figure 4 illustrates the difference maps of the six VOCs at four different concentrations. To demonstrate the effectiveness of the sensor clearly, the initial RGB value was amplified ten-fold. The results show significant crosswise differences among the difference maps of the analytes, where targets with similar structures have similar regional results; slight differences occur among the results for the same analyte at different concentrations longitudinally, with a positive correlation reflecting the increase in the response signals of the difference maps. This study has thus realized effective identification of multiple lung cancer markers in exhaled breath and the 
detected concentrations can reach the ppb level; this is intended to provide solid technical support for more comprehensive detection of these lung cancer markers.

\subsection{Response data analysis of the VOCs}

The data were analyzed using direct digital image acquisition technology, and each analyte was described using a 30-dimensional vector (RGB differences of 10 sensitive substances). The Euclidean distance (ED) is generated using equations with the following formula: $\mathrm{ED}=\operatorname{sqrt}\left[\left(\mathrm{R}_{1}-\mathrm{R}_{1}{ }^{0}\right)^{2}+\left(\mathrm{G}_{1}-\mathrm{G}_{1}{ }^{0}\right)^{2}+\left(\mathrm{B}_{1}-\mathrm{B}_{1}{ }^{0}\right)^{2}+\ldots\left(\mathrm{R}_{10}-\mathrm{R}_{10}{ }^{0}\right)^{2}+\left(\mathrm{G}_{10}-\mathrm{G}_{10}{ }^{0}\right)^{2}+\left(\mathrm{B}_{10}-\mathrm{B}_{10}{ }^{0}\right)^{2}\right] . \quad$ The Euclidean distance-response time curve (Fig. 5) shows that the equilibrium time for the six VOCs is $6 \mathrm{~min}$; the Euclidean distance of an analyte containing an active group was longer than that of a relatively stable analyte, e.g., the Euclidean distances of benzenes and 1,2,4-trimethylbenzene were longer than the corresponding distances for decane and undecane. The detection results for the $\mathrm{N}_{2}$ control group (Euclidean distance of close to 0, RSD value of $0.1 \%$ ) shows that the system has weak interference and high stability.

\subsection{Pattern recognition analysis of VOCs}

Principal component analysis (PCA) is performed to visualize the data, and the two main component score vectors are used to draw the two-dimensional scatter diagram for the five times parallel data of the six VOCs at $10 \mathrm{ppm}$. As illustrated in Fig. 6(a), the two components that account for $75.2 \%$ of the total amount of information can be used to separate the analytes, and there is no overlap among scatter plots for all samples. When the parallel samples are aggregated together, the analytes with similar structures are close to each other, while the different structural analytes are located far apart. Hierarchical cluster analysis (HCA) is a process in which an abstract analyte is separated into similar analytes. The response system tree obtained in this study represents the multivariate distances among the analytes in the 30-dimensional RGB color space. The HCA tree diagram illustrates that the detection results from the parallel experiment initially aggregate into a cluster; classification of the analytes matches the structural similarity pattern of the VOCs; the detection objects are then classified into different sets according to their similarity (Fig. 6(b)). The illustrations visually indicate the specific response areas of the three VOCs.

\section{Discussion}

This research is based on combination of the cross-response principle with the pattern recognition method to achieve a characteristic fingerprint. The pattern recognition algorithm can 
obtain the properties, concentration and similarity of the object. The independently constructed dual-channel microarray sensor system offers the advantages of rapid noninvasive testing at low cost and with high sensitivity, and is thus an ideal screening method for major diseases such as lung cancer. When compared with the single-channel sensing method, it is easier to realize multiple real-time controlled experiments and double-sample synchronization parallel experiments using the proposed system, which effectively improves the experimental accuracy and the detection speed. The study successfully achieved rapid, high-sensitivity detection of most representative lung cancer markers, and the results of analysis of the differential spectra, HCA and PCA consistently showed that the classification of the analytes conformed to the law of structural similarity, which is intended to provide an important guarantee for effective detection of the clinical breath samples at a later stage.

\subsection{Optimization effects of the sensor}

When lung cancer occurs, lipid peroxidation takes place in the unsaturated fatty acid on the cell membrane or on the subcell membrane and generates alkanes that are difficult to dissolve or metabolize in blood and are excreted out via expiration; aldehydes are generated during the detoxification process of cytochrome p450 and the metabolism of tobacco; and aromatics are generated from the effects of smoking, drinking, pollution and radiation, causing peroxidation damage to unsaturated fatty acids on the cell membranes, protein and DNA. Lung cancer-related VOCs are released slowly via excretion in relatively high concentrations. For the most widely accepted three types of VOC described above, ten types of sensitive material were screened precisely to construct a $2 \times 5$ microarray for sensing of six VOCs at $10 \mathrm{ppm}$. The sensitivity $\mathrm{S}=\triangle \mathrm{E} \div \mathrm{C}$, where $\Delta \mathrm{E}$ is the Euclidean distance difference between the sample and the blank; and $\mathrm{C}$ is the concentration of the detected substance. In this case, a larger $\mathrm{S}$ value indicates higher sensitivity. Comparison of the optimized method (dual channel $2 \times 5$ microarray) and the primary method (single channel $6 \times 6$ array) confirmed the feasibility and validity of the proposed method. The results (Fig. 7) show that the sensitivity and the stability of the optimized method increased by approximately 1.3 times for the microchannels constructed by soft lithography in microfluidic devices, while the detection speed increased by two times, and the workload, cost and production time for the chips were reduced by two-thirds for two-channel high speed data acquisition system and well-screened photosensitive materials. Therefore, this study reduces the detection cost and 
the corresponding workload while also effectively improving the detection sensitivity and stability and the detection speed.

\subsection{Cross-response mechanism of the sensor}

The dual-channel microarray sensor established in this work showed good stability and sensitivity, which is attributed to the molecular interactions that occur between the sensitive materials and the target materials on the sensor array (Fig. 8); the sensitive materials that contain hydroxyl may form hydrogen bonds with the aldehydes; the sensitive materials that contain benzene rings may form $\pi-\pi$ interactions with the aromatics; acid-base indicators may have Lewis weak acid base reactions with aldehydes/aromatics on the surface; porphyrins may form metal coordinations with the VOCs; and the sensitive materials may have Van der Waals' force and physical adsorption interactions with the target molecules. These interactions realize the crossresponses for the VOCs in a class-selective manner via synergetic effects. Compared with other techniques like electrochemical method, ion mobility spectrometry and so on, the visualized microfluidic channels method constructed in this paper was a new attempt and was of the characteristics of intuition, simplicity, celerity, accuracy and little interference.

In this study, the Tenax Ta $200 \mathrm{mg}$ enrichment device, the ATDS analyzer and the dualchannel microarray sensor are integrated together to realize purification and enrichment/thermal desorption/sensing detection for multicomponent samples, which can effectively avoid generation of interference signals and missed detection signals while improving the accuracy of the sensing analysis. Optical materials with stable performances, high sensitivity, active centers and chromophores have been accurately screened as the sensing units of the microarrays to improve their selectivity. The dual-channel microarray sensor was constructed to realize real-time contrast detection and synchronous parallel detection, thus enhancing the recognition ability of the sensor system. This study provides an important guarantee and a strategy for noninvasive, rapid, economical and efficient lung cancer screening, and has potential application prospects for both extensive lung cancer screenings and large-scale health screenings.

\section{Conclusions}

In this paper, a prototype of a highly sensitive and selective dual-channel microarray sensor based on porphyrins and dyes has been fabricated for qualitative and semi-quantitative analysis of analytes. In this research, a series of color difference maps for six analytes were obtained 
precisely, and the RGB response signals were further analyzed using PCA and HCA. The analysis results showed that the microarray sensor provided a rapid response within 6 min for VOC identification with high sensitivity; this is possible because the sensing materials have high affinity and selectivity for the analytes. The dual channel microarray sensor's speediness and high sensitivity mean that the noninvasive technique will be suitable for lung cancer screening and other types of large-scale health screening.

\section{Acknowledgements}

Funding: This work was supported by the National Natural Science Foundation of China [grant numbers NSFC81703569, 81870005 to RL, NSFC31770613 to JJ]; the Project supported by the Natural Science Foundation of the Jiangsu Higher Education Institutions of China [grant numbers 18KJB220004 to YX, 18KJA180003 to RL]; the Project of Xuzhou Applied and Basic Research [grant number KC18009 to RL]; the Doctorate Teacher Support Project of Jiangsu Normal University [grant number 15XLR027]; Postgraduate Research \& Practice Innovation Program of Jiangsu Province [KYCX18-2142 to PZ]; the Priority Academic Program Development of Jiangsu Higher Education Institutions (PAPD) and the Sharing Fund for Jiangsu Normal University's large equipment.

\section{References}

[1] F. Bray, J. Ferlay, I. Soerjomataram, R.L. Siegel, L.A. Torre, A. Jemal, Global cancer statistics 2018: GLOBOCAN estimates of incidence and mortality worldwide for 36 cancers in 185 countries, CA Cancer J Clin 68 (2018) 394-424.

[2] R.F. Machado, D. Laskowski, O. Deffenderfer, T. Burch, S. Zheng, P.J. Mazzone, T. Mekhail, C. Jennings, J.K. Stoller, J. Pyle, J. Duncan, R.A. Dweik, S.C. Erzurum, Detection of lung cancer by sensor array analyses of exhaled breath, Am J Respir Crit Care Med 171 (2005) 1286-1291.

[3] F.L. Jacobson, M.T. Jaklitsch, Computed Tomography Scanning for Early Detection of Lung Cancer, Annu Rev Med 69 (2018) 235-245.

[4] M. Treskova, I. Aumann, H. Golpon, J. Vogel-Claussen, T. Welte, A. Kuhlmann, Trade-off between benefits, harms and economic efficiency of low-dose CT lung cancer screening: a microsimulation analysis of nodule management strategies in a population-based setting, BMC Med 15 (2017) 162.

[5] G. Rocco, G. Pennazza, M. Santonico, F. Longo, R. Rocco, P. Crucitti, R. Antonelli Incalzi, Breathprinting and Early Diagnosis of Lung Cancer, J Thorac Oncol 13 (2018) 883-894.

[6] L. Pauling, A.B. Robinson, R. Teranishi, P. Cary, Quantitative analysis of urine vapor and breath by gas-liquid partition chromatography, Proc Natl Acad Sci U S A 68 (1971) 2374-2376.

[7] Y. Bao, P. Xu, S. Cai, H. Yu, X. Li, Detection of volatile-organic-compounds (VOCs) in solution using cantilever-based gas sensors, Talanta 182 (2018) 148-155.

[8] R. Gasparri, M. Santonico, C. Valentini, G. Sedda, A. Borri, F. Petrella, P. Maisonneuve, G. Pennazza, A. D'Amico, C. Di Natale, R. Paolesse, L. Spaggiari, Volatile signature for the early 
diagnosis of lung cancer, J Breath Res 10 (2016) 016007.

[9] J. Zhou, Z.A. Huang, U. Kumar, D.D.Y. Chen, Review of recent developments in determining volatile organic compounds in exhaled breath as biomarkers for lung cancer diagnosis, Anal Chim Acta 996 (2017) 1-9.

[10] F. Mottaghitalab, M. Farokhi, Y. Fatahi, F. Atyabi, R. Dinarvand, New insights into designing hybrid nanoparticles for lung cancer: Diagnosis and treatment, J Control Release 295 (2019) 250-267.

[11] H. Haick, Y.Y. Broza, P. Mochalski, V. Ruzsanyi, A. Amann, Assessment, origin, and implementation of breath volatile cancer markers, Chem Soc Rev 43 (2014) 1423-1449.

[12] P.J. Mazzone, J. Jett, Principled Lung Cancer Screening Follows Screening Principles, Chest 154 (2018) 1265-1266.

[13] J.E. Chang, D.S. Lee, S.W. Ban, J. Oh, M.Y. Jung, S.H. Kim, S. Park, K. Persaud, S. Jheon, Analysis of volatile organic compounds in exhaled breath for lung cancer diagnosis using a sensor system, Sensor Actuat B-Chem 255 (2018) 800-807.

[14] R. Ehmann, E. Boedeker, U. Friedrich, J. Sagert, J. Dippon, G. Friedel, T. Walles, Canine scent detection in the diagnosis of lung cancer: revisiting a puzzling phenomenon, Eur Respir J 39 (2012) 669-676.

[15] G. Peng, U. Tisch, O. Adams, M. Hakim, N. Shehada, Y.Y. Broza, S. Billan, R. Abdah-Bortnyak, A. Kuten, H. Haick, Diagnosing lung cancer in exhaled breath using gold nanoparticles, Nat Nanotechnol 4 (2009) 669-673.

[16] Y. Adiguzel, H. Kulah, Breath sensors for lung cancer diagnosis, Biosens Bioelectron 65 (2015) 121-138.

[17] R. Capuano, A. Catini, R. Paolesse, C. Di Natale, Sensors for Lung Cancer Diagnosis, J Clin Med 8 (2019).

[18] N.A. Rakow, K.S. Suslick, A colorimetric sensor array for odour visualization, Nature 406 (2000) 710-713.

[19] P.J. Mazzone, X.F. Wang, Y.M. Xu, T. Mekhail, M.C. Beukemann, J. Na, J.W. Kemling, K.S. Suslick, M. Sasidhar, Exhaled Breath Analysis with a Colorimetric Sensor Array for the Identification and Characterization of Lung Cancer, Journal of Thoracic Oncology 7 (2012) 137-142.

[20] D.Q. Huo, Y.H. Xu, C.J. Hou, M. Yang, H.B. Fa, A novel optical chemical sensor based AuNRMTPP and dyes for lung cancer biomarkers in exhaled breath identification, Sensor Actuat B-Chem 199 (2014) 446-456.

\section{Tables Captions}


Table 1 Calculation formulas for $\mathrm{V}_{\mathrm{i}}$ and RSD

Table 2 RSD and $\mathrm{V}_{\mathrm{i}}$ of 10ppm VOCs and $\mathrm{N}_{2}$ 


\begin{tabular}{|c|c|}
\hline Name & Calculation formulas \\
\hline & Antoine equation: $\lg \mathrm{P}_{\mathrm{i}}=\mathrm{A}-\mathrm{B} /(\mathrm{t}+\mathrm{C})$ \\
$\mathrm{V}_{\mathrm{i}}$ & $\mathrm{C}_{\mathrm{i}}=\mathrm{P}_{\mathrm{i}} / \mathrm{RT} \times 22.4 / \mathrm{M} \times(273+\mathrm{t}) / 273$ \\
& $\mathrm{~V}_{\mathrm{i}}=\mathrm{C}_{\mathrm{s}} / \mathrm{C}_{\mathrm{i}} \times \mathrm{V}_{\mathrm{s}}$ \\
\hline $\mathrm{RSD}$ & $S=\sqrt{\frac{\sum_{i=1}^{n}\left(x_{\mathrm{i}}-\bar{x}\right)^{2}}{n-1}}$ \\
& $R S D=\frac{S}{\bar{x}} \times 100 \%$ \\
\hline
\end{tabular}

$\mathrm{A}, \mathrm{B}, \mathrm{C}$ : Antoine parameters; $\mathrm{C}$ : gas concentration of saturated vapor, ppm; $\mathrm{t}$ : detection temperature, ${ }^{\circ} \mathrm{C} ; \mathrm{V}_{\mathrm{i}}$ : detected volume of saturated vapor, $\mathrm{mL}$; $\mathrm{C}_{\mathrm{s}}$ : detected concentration, $10 \mathrm{ppm}$; $\mathrm{V}_{\mathrm{s}}: 10 \mathrm{~L}$; RSD: relative standard deviation; $\quad \mathrm{X}$ : average value; S: standard deviation. 
Table 2

\begin{tabular}{cccccc}
\hline Category & Name & Pi & T/K & Vi/mL & $\%$ RSD \\
\hline Alkanes & decane & 150 & 293 & 16.7 & 2.0 \\
& undecane & 140 & 293 & 17.8 & 2.1 \\
Aromatic compounds & benzene & 13820 & 300 & 0.2 & 1.8 \\
& 1,2,4-trimethybenzene & 280 & 293 & 9.0 & 2.9 \\
Aldehydes & hexanal & 1150 & 293 & 2.2 & 1.7 \\
heptanal & 400 & 298 & 6.3 & 2.2 \\
Control group & $\mathrm{N}_{2}$ & & 298 & 10 & 0.1 \\
\hline
\end{tabular}




\section{Figures Captions}

Fig. 1. Three sensitive material types for microarray construction.

Fig. 2. Optical control-signal acquisition system for microarray sensor and its working principle (a-CCD camera; b-microarray; c-LED light source; d-computer; e-initial image; f-final image; gdifference map).

Fig. 3. Enrichment and thermal desorption processes for dual-channel microarray sensing system (a-Tenax Ta $200 \mathrm{mg}$ enrichment device; b-ATDS-3400B thermal desorption device; c-Dualchannel microarray sensor device; d-Computer).

Fig. 4. Digital color difference maps of the sensor array for six VOCs at four concentrations.

Fig. 5. Euclidean distance-response time curves for six VOCs at $10 \mathrm{ppm}$.

Fig. 6. (a) Scatter diagram of PCA, and (b) dendrogram of HCA for six VOCs at $10 \mathrm{ppm}$; the corresponding difference maps are shown in the inset.

Fig. 7. (a) Euclidean distance and (b) digital color difference maps of the optimized method and the primary method for six VOCs at $10 \mathrm{ppm}$.

Fig. 8. Possible microarray recognition mechanism for analytes. 
Fig. 1.
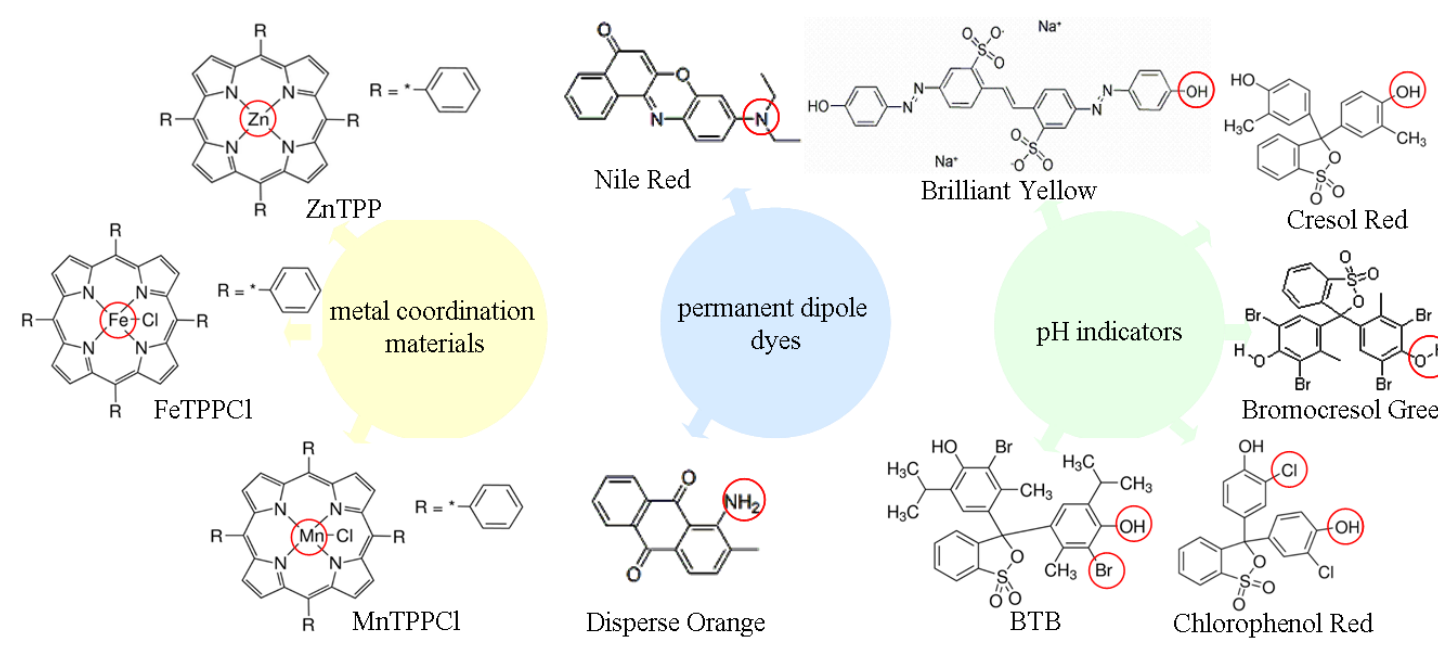

permanent dipole dyes
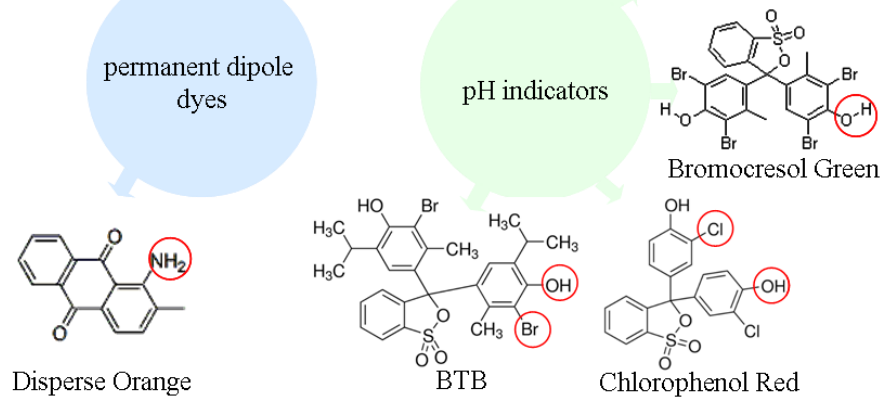
Fig. 2.

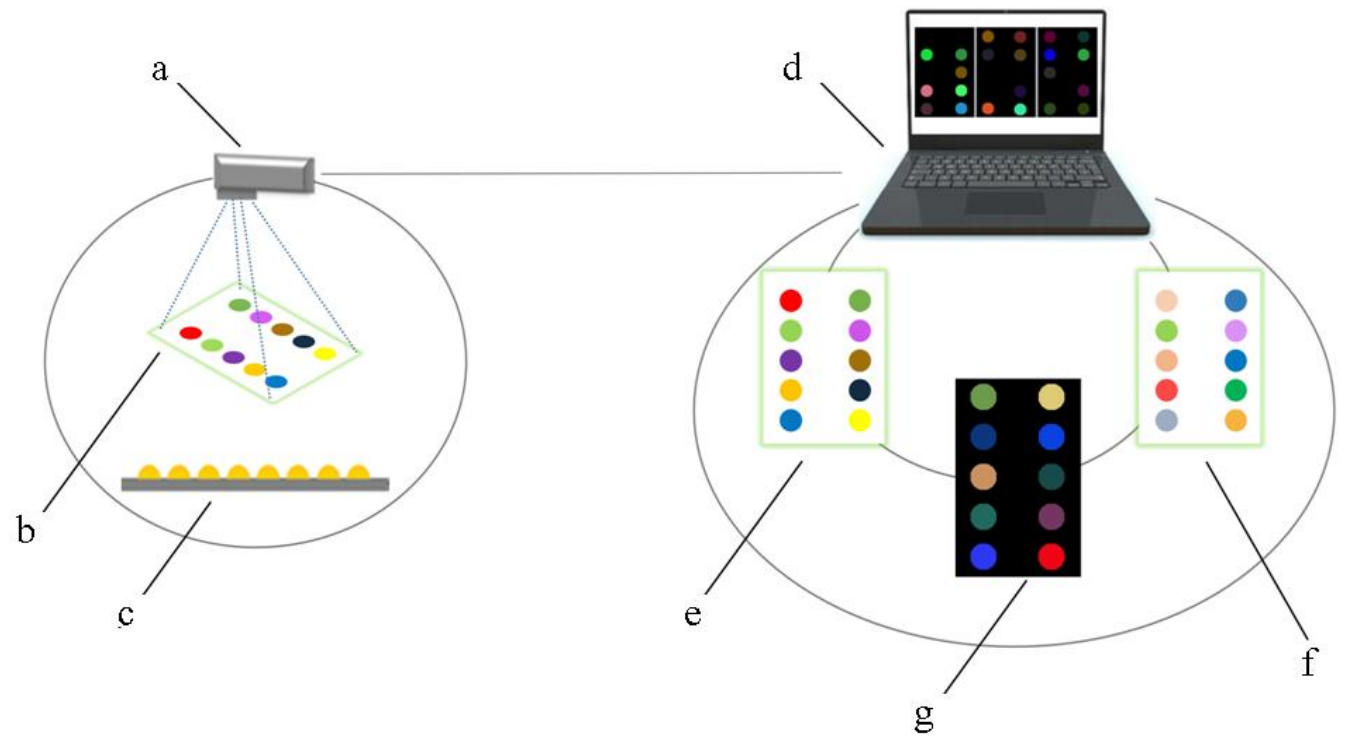


Fig. 3.

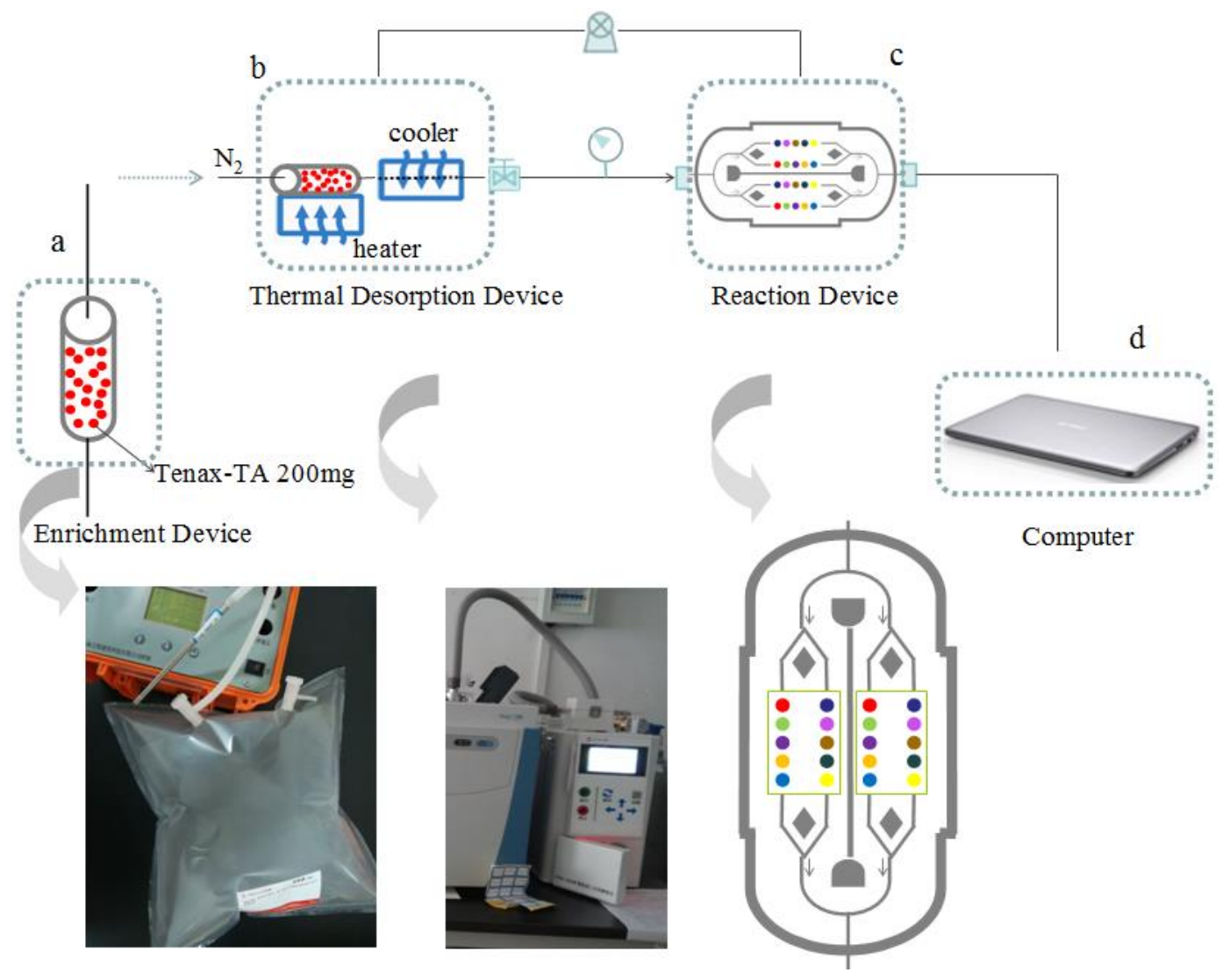


Fig. 4. 


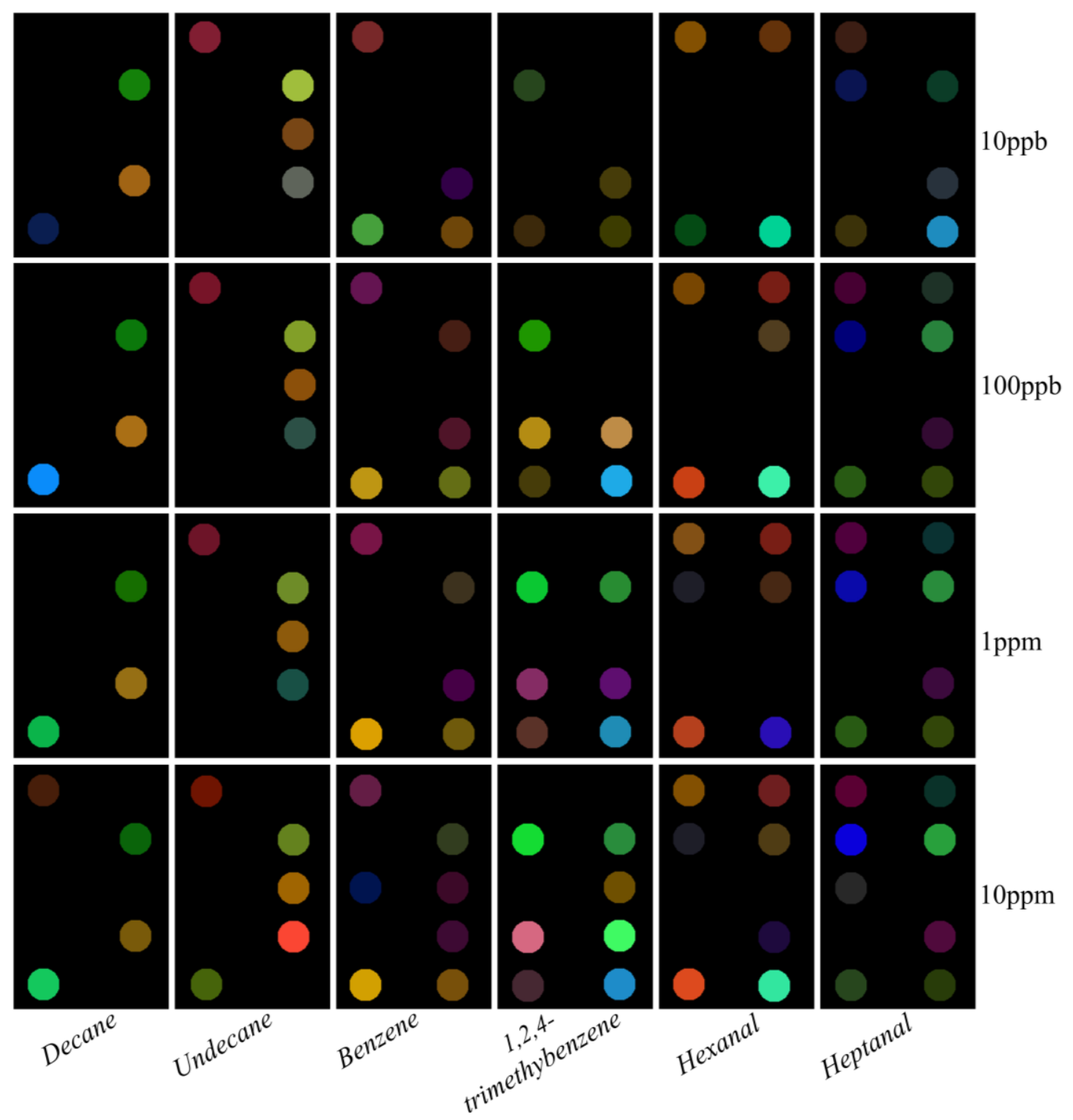

Fig. 5. 

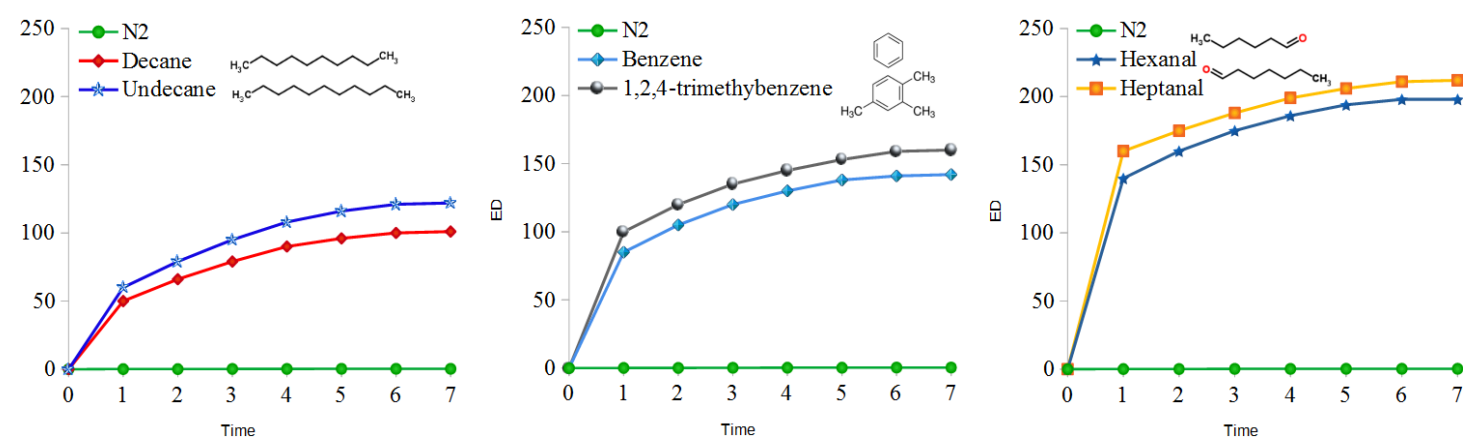
Fig. 6 .

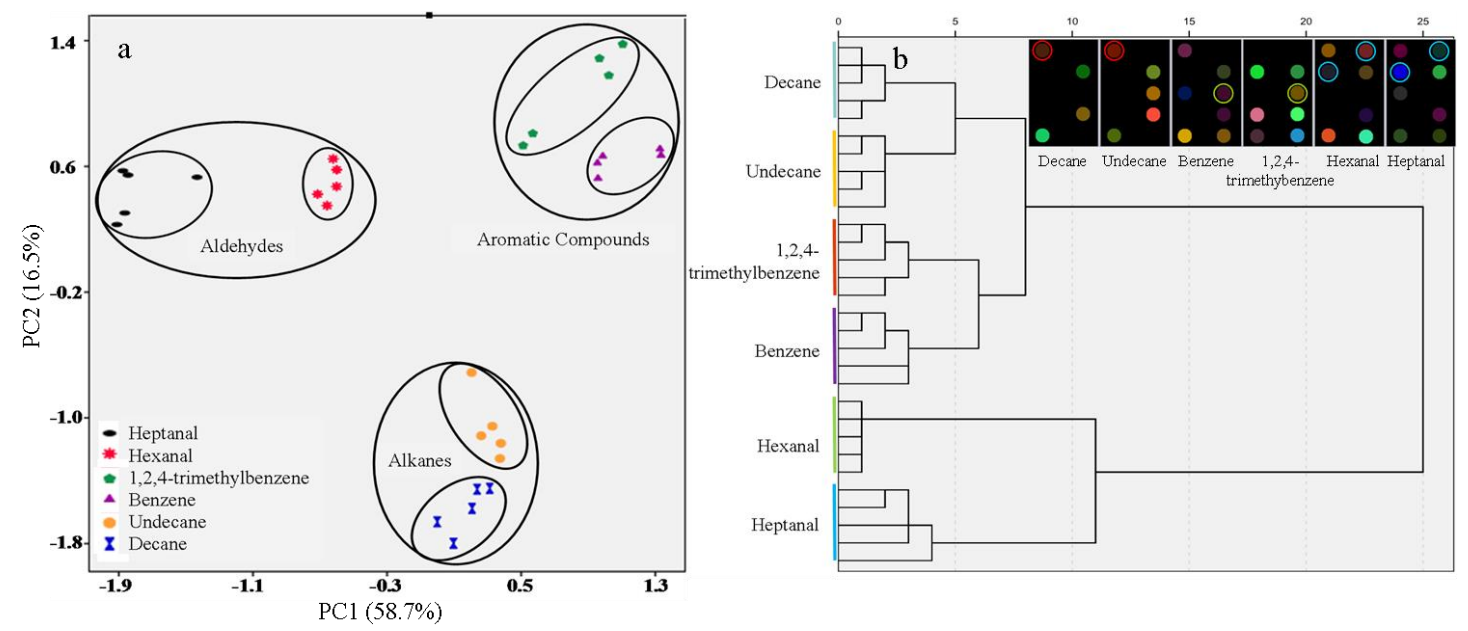


Fig. 7.

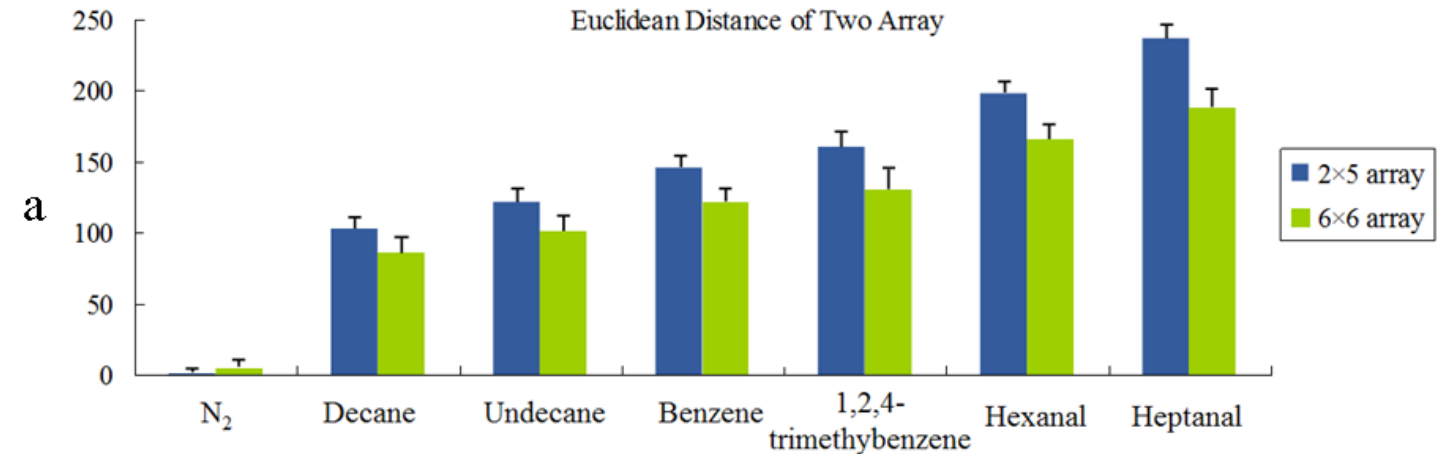

b

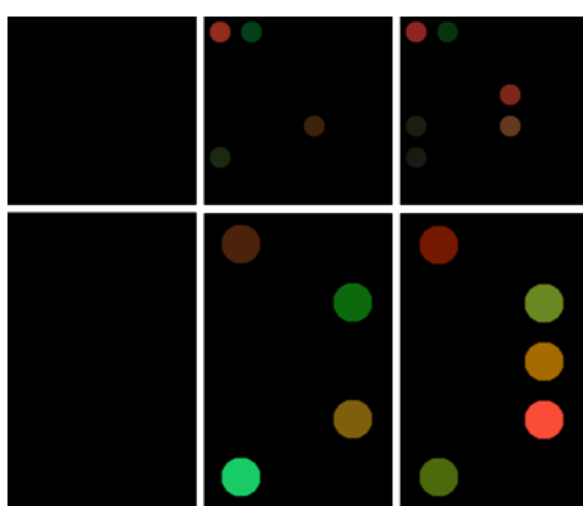

$\mathrm{N}_{2}$

Decane

Undecane

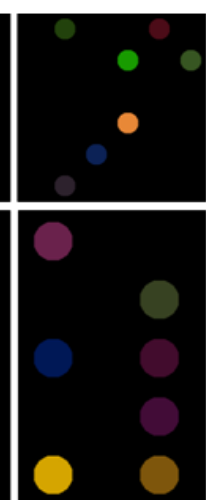

Benzene

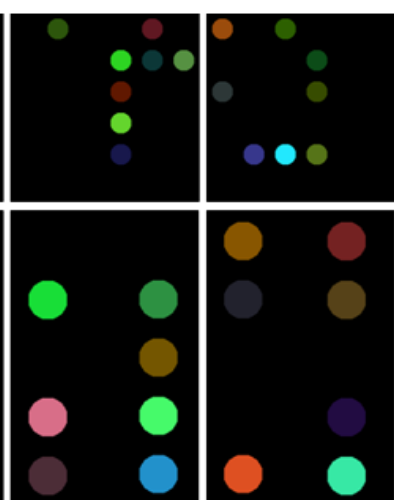

$1,2,4-$

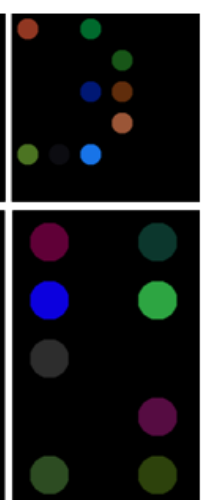

Heptanal trimethybenzene 
Fig. 8 .

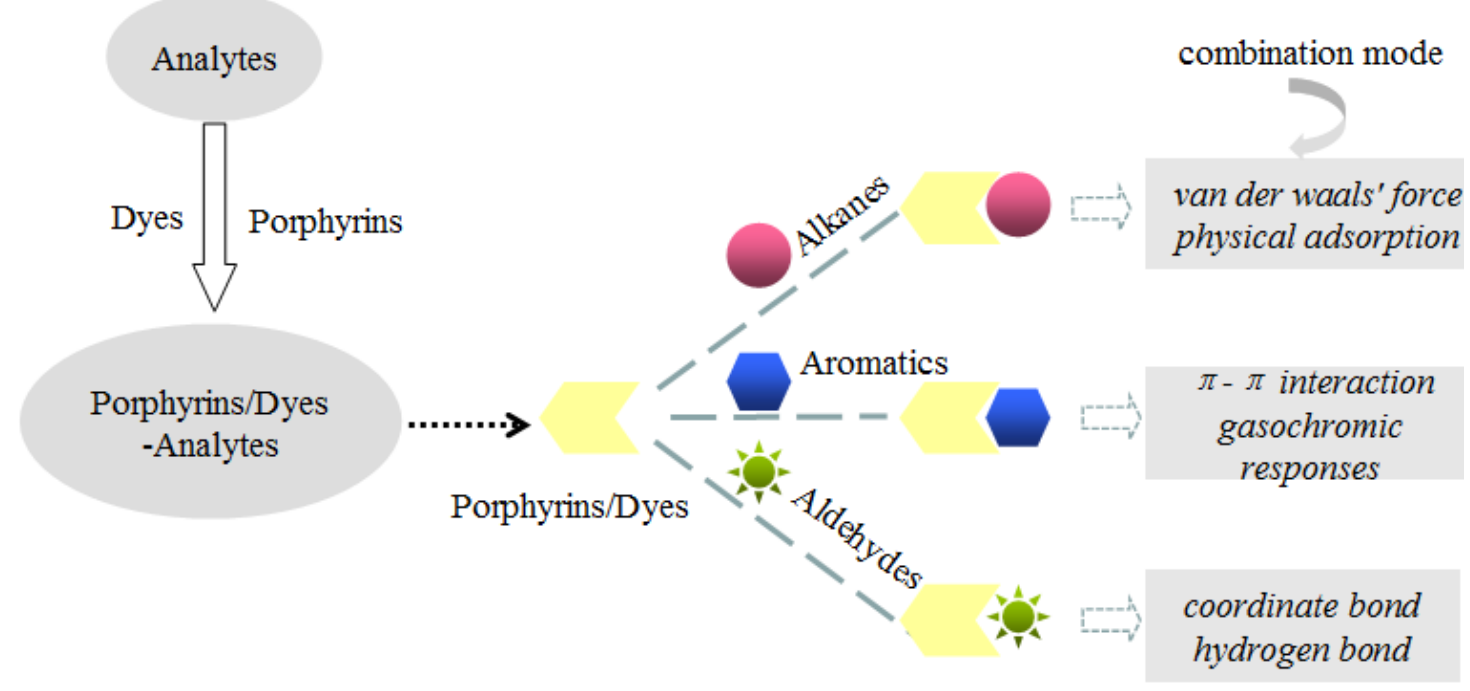




\section{Biographies}

Rongpeng Li received B.S. degree in Life Sciences from Nanjing Normal University (NJNU) in 2005, Ph.D. degree in Microbiology from NJNU in 2012. He received his Postdoctoral trainings from Nanjing University of Technology in 2012.07-2014.03, and from University of North Dakota (USA) from 2014.03-2017.04. He is currently professor of the Key Laboratory of Biotechnology for Medicinal Plant of Jiangsu Province in Jiangsu Normal University. He has published more than 30 academic papers in journals. His research interests include: macrophage function (phagosome maturation) relating to signaling molecules including TLRs, Lyn, annexin A2, caveolin 1, and lipid rafts, and how epigenetic modifications (miRNAs) are associated with pathogenesis of lung infection or other diseases (asthma, chronic obstructive pulmonary disease, and cancer).

Yonghong Xu obtained her M.Ed. degree in chemical education from Xinjiang Normal University in 2010, Ph.D. degree in Biomedical Engineering (BME) from Chongqing University in 2015. She is currently lecturer of the Key Laboratory of Biotechnology for Medicinal Plant of Jiangsu Province in Jiangsu Normal University. She has published about 10 academic papers. Her research interests involve sensing materials, biochip, biochemical sensor, microsystem and non-invasive diagnosis technique for lung cancer.

Jihong Jiang received his Ph.D. degree in forest resources protection from Nanjing Forestry University in 1997. He is currently professor of the Key Laboratory of Biotechnology for Medicinal Plant of Jiangsu Province in Jiangsu Normal University. He has published more than 70 academic papers during the past five years and he has obtained more than 10 national invention patents. His research interests include: microbial ecology, medical biological resources and biotechnology, rare medicinal and edible resources and endophytic bacteria resources and their 
functions. 